\title{
Catchment approach to passive sampling of Irish waters
}

Lisa Jones, J. Ronan, B. McHugh, F. Regan May 21'st 2015

Oceans'15 


\section{Outline}

- Project description

- Sampling sites

- Passive sampling

- Cork catchment

- Overview

- Results

- Time trial

- Dublin catchment

- Results

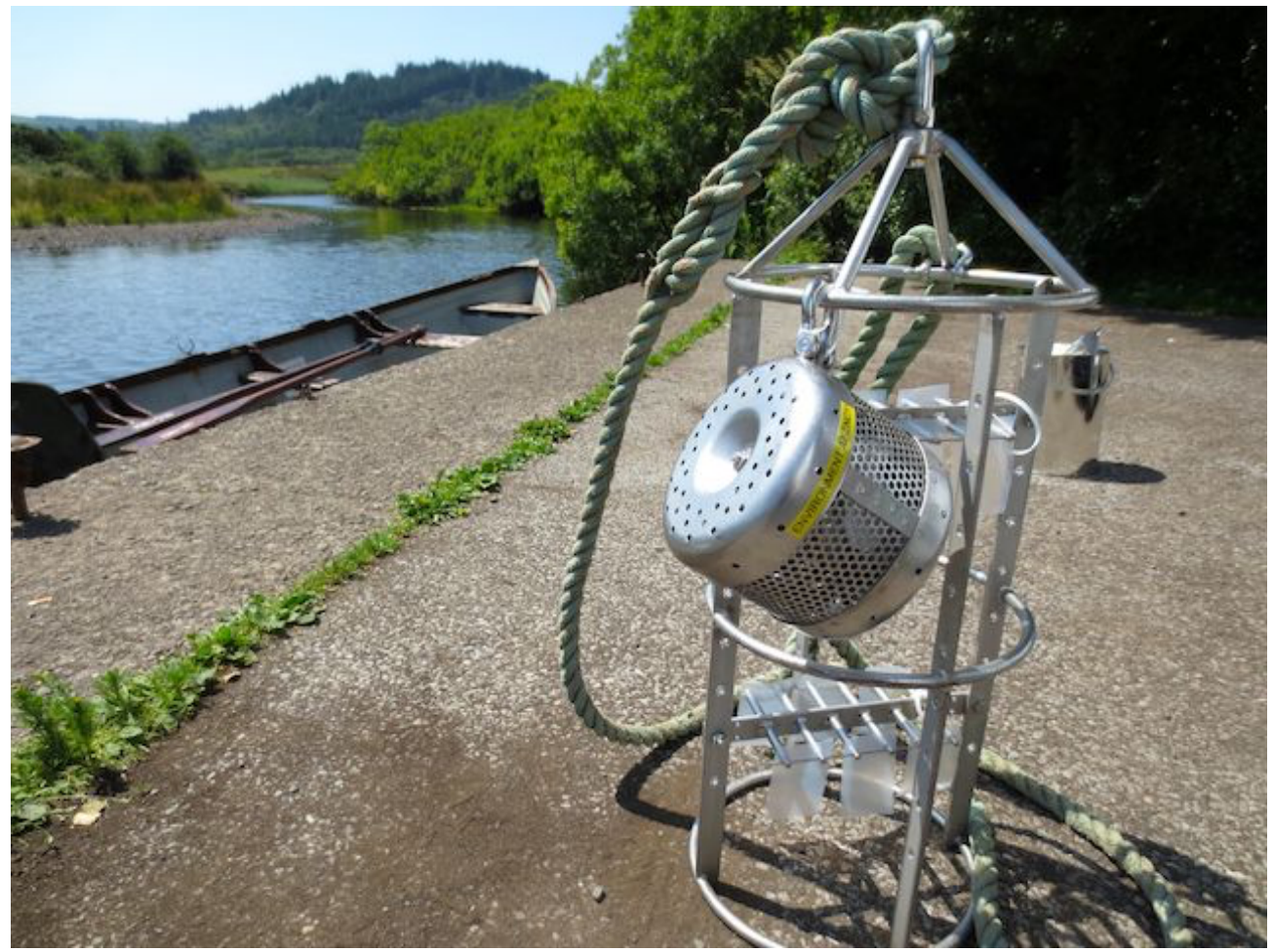

- Conclusions and outputs 


\section{Project description}

- EPA funded 3 year project

- Role of PS as a screening and monitoring tool for new and emerging chemicals

- Role of PS as a surrogate to biota

- Qualitative/quantitative screening of selected substances in a number of Irish waters representative of different pressures

- Case studies on emerging compounds and pharmaceuticals using a catchment approach 


\section{Passive Sampling}

- Determination of pollutants in aquatic environment

- Free flow of analyte molecules from sampled medium to collecting medium - only dissolved analytes, no energy source

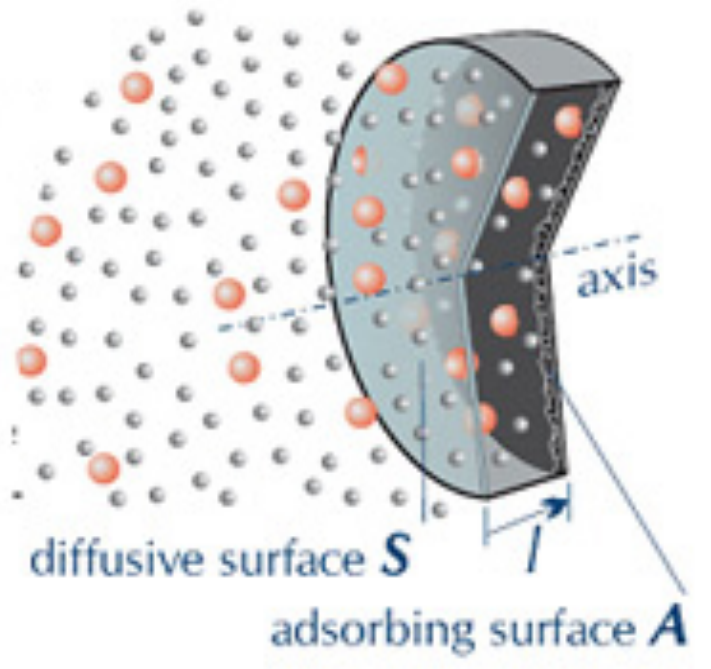

Fig. 1 - Passive sampling mechanism

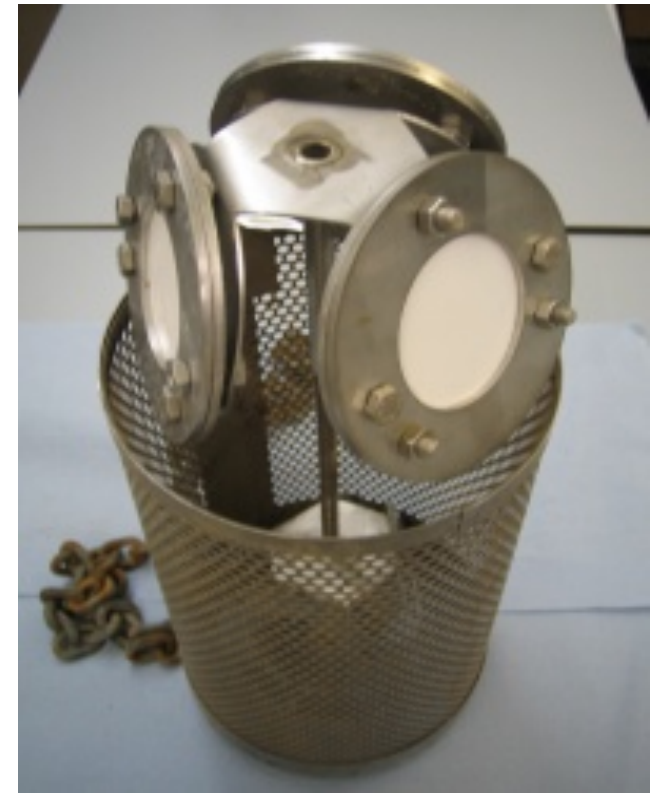

Fig. 2 - Passive sampling device (interior)

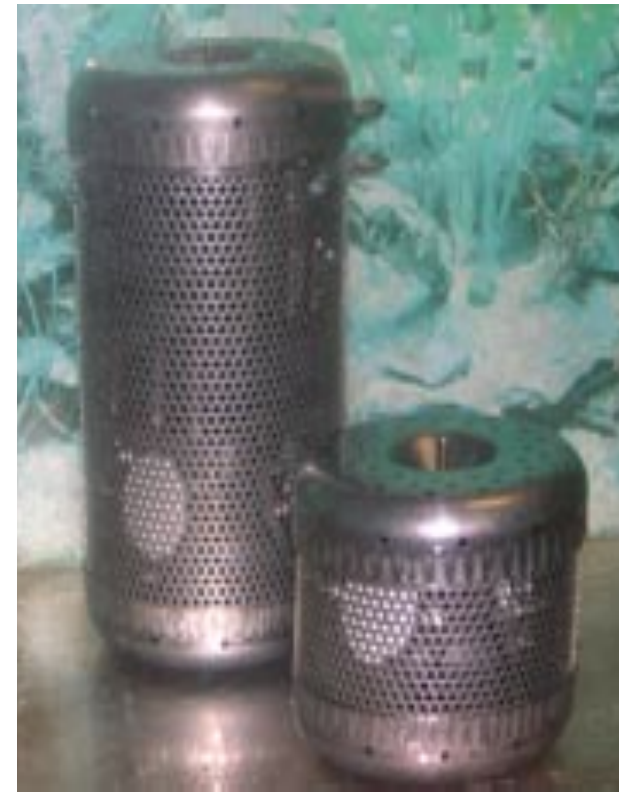

Fig. 3 - Passive sampling devices 


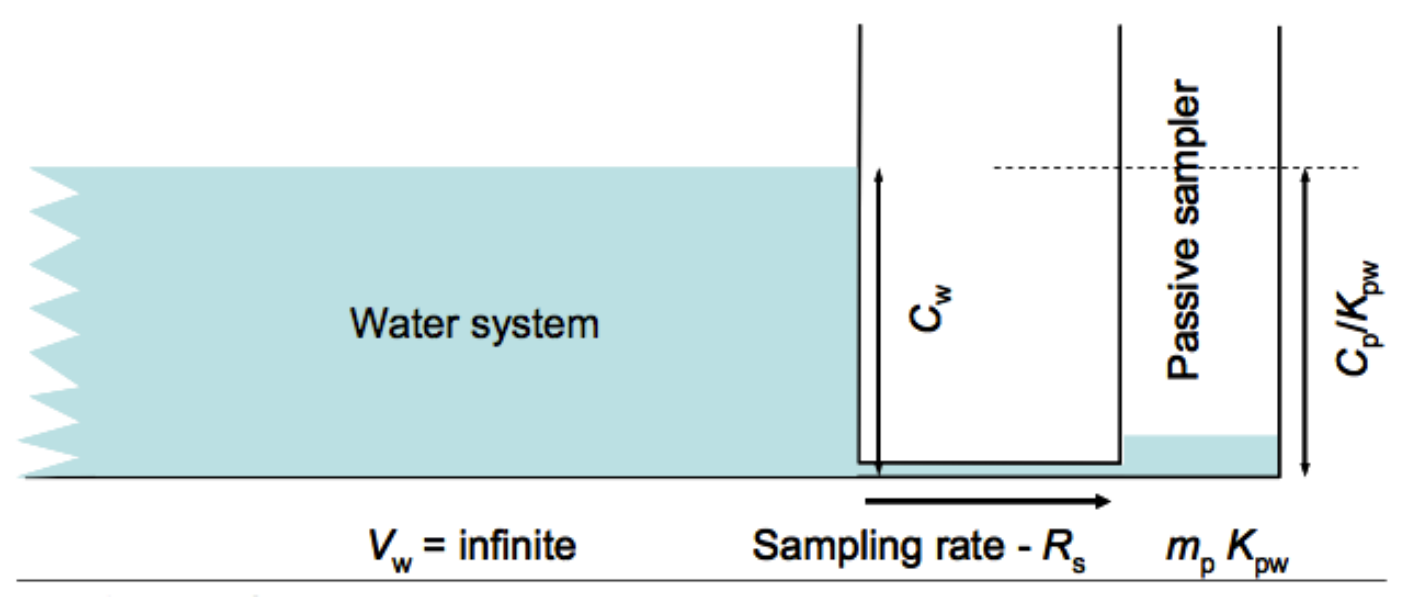

Fig. 4 - Absorption passive sampling mechanism

Equilibrium is reached and time-weighted average is determined. Mainly for nonpolar compounds.

Fig. 5 - Adsorption passive sampling mechanism

Kinetic regime is maintained and calculations are based on time-integrated measurements Mainly for polar analytes.

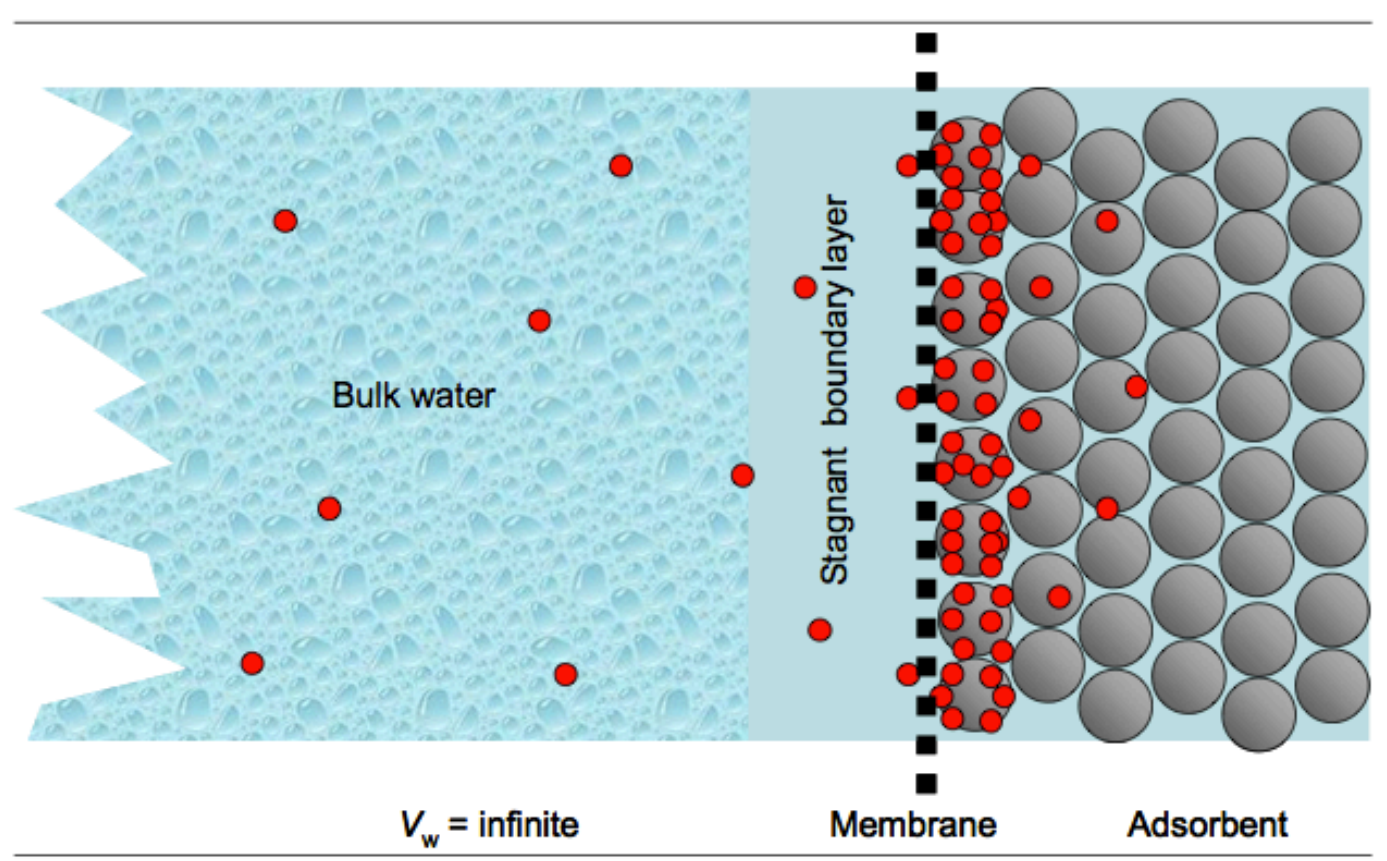

Ref: Smedes et al., 2010 


\section{Passive Sampling}

- Greater sensitivity than can be achieved by "traditional" spot-sampling

- Applicable to a wide variety of compounds

- Time-integrated sampling at low detection limits and in-situ extraction of analytes

- Ability to sample large volumes of water

- Ease of deployment and processing

- No external power input is required 


\section{Protocol for Passive Sampler Deployment}

- ICES TIMES no. 52* for PDMS

- EA lab/NLS guidelines for POCIS

Record

- GPS co-ordinates

- Date and time of deployment

- Salinity

- Water temperature

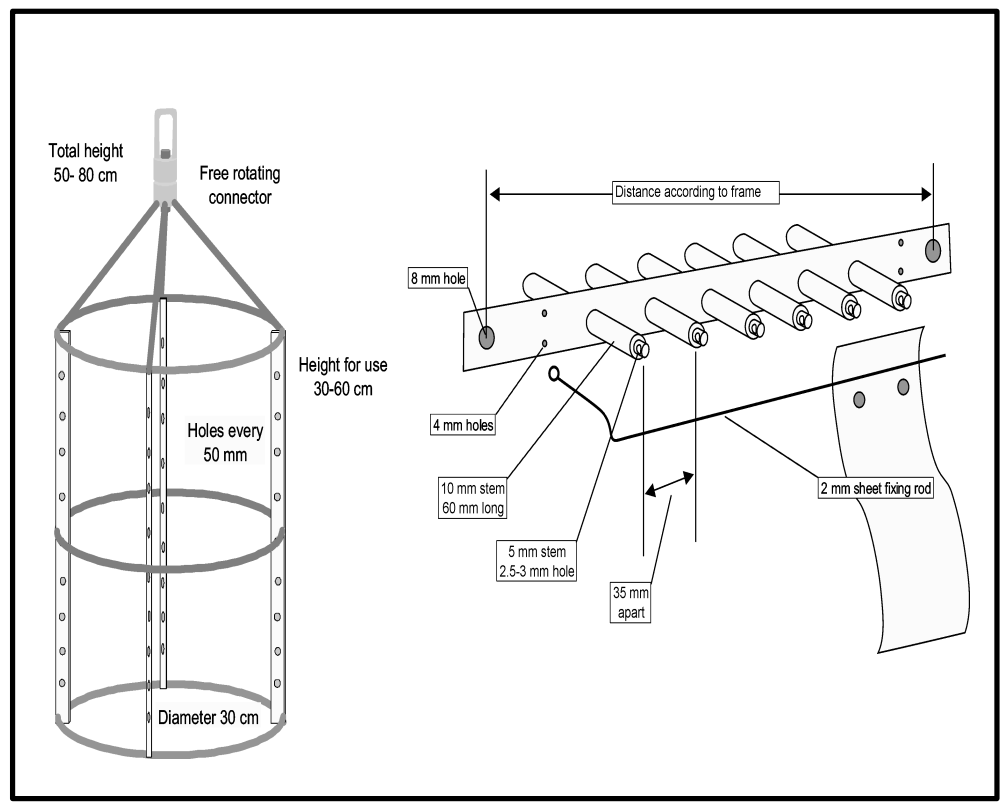

PDMS sheet attachment*

*ICES TIMES no. 52. 2012. Guidelines for passive sampling of hydrophobic contaminants in water using silicone rubber

**Environmental Sampling Technologies lab: http://www.est-lab.com/pocis.php

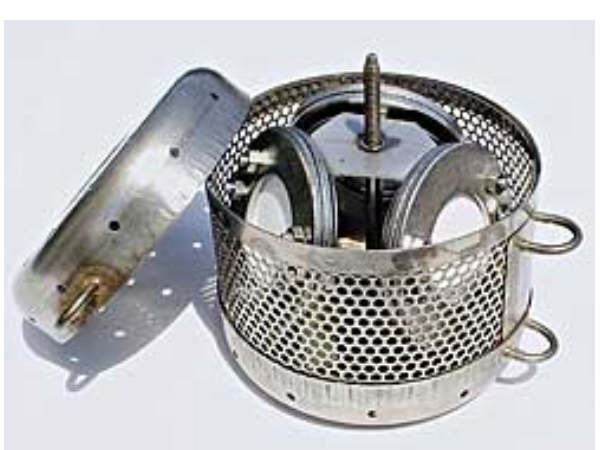

POCIS canister** 


\section{IRISH PERSPECTIVE}




\section{Status of Irish Water}

- 31 of the 41 WFD Priority Substances and 89 of the 161 relevant pollutants were detected in one or more samples.

The most commonly detected compounds were metals and polycyclic aromatic hydrocarbon (PAH). 


\section{Irish Rivers}

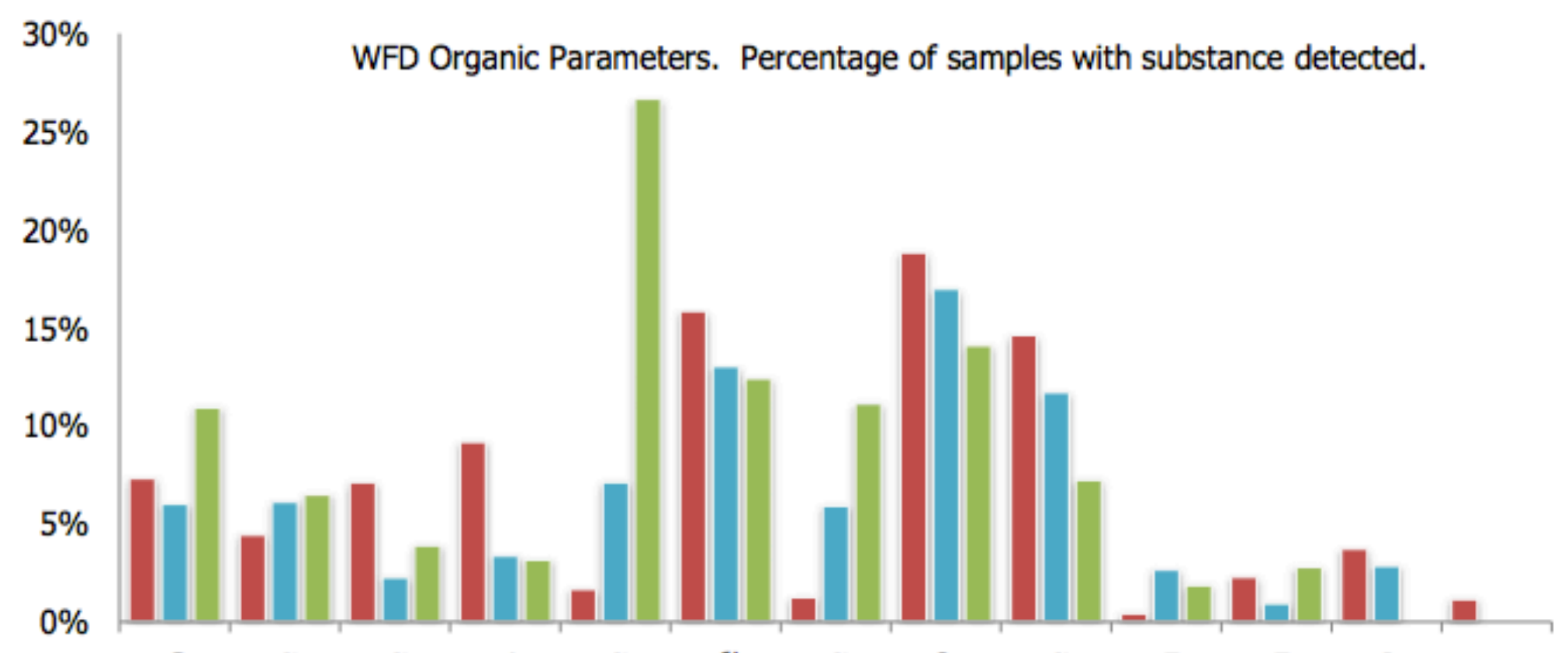

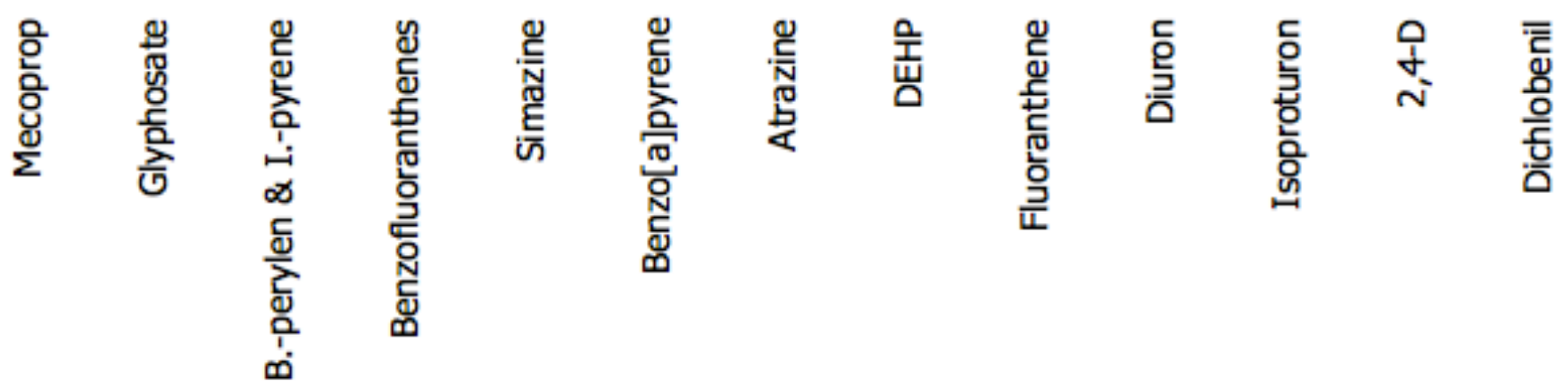

$$
\text { = } 2009>=\mathrm{LOQ} \quad=2008>=\mathrm{LOQ} \quad=2007>=\mathrm{LOQ}
$$




\section{Irish Rivers}

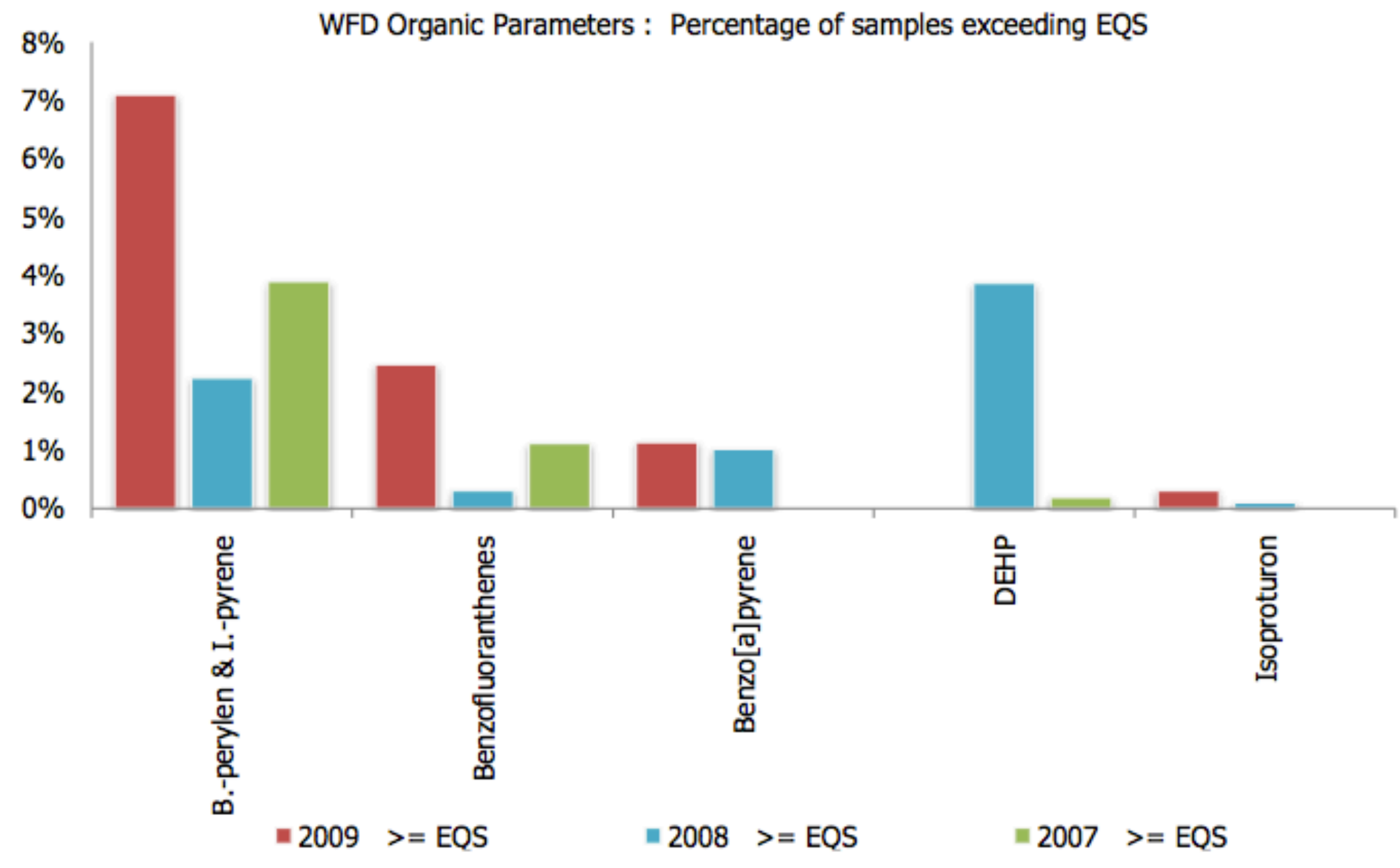

(EPA Water Quality Report, 2010) 
Overview of Irish agencies with potential information relating to priority substances in Irish waters

\begin{tabular}{|c|c|c|c|c|c|}
\hline & EPA & RBDs & DAFF & LAs & Other (14 Agencies) \\
\hline Surface water & $\checkmark$ & $\checkmark$ & & $\checkmark$ & 4 others \\
\hline Groundwater & $\checkmark$ & $\checkmark$ & & $\checkmark$ & 4 others \\
\hline Landfill & $\checkmark$ & & & $\checkmark$ & \\
\hline Mining & $\checkmark$ & & & & \\
\hline Stormwater/runoff & & & & & 1 other \\
\hline WWTPS & $\checkmark$ & & & $\checkmark$ & \\
\hline Industry & $\checkmark$ & & $\checkmark$ & $\checkmark$ & \\
\hline Agriculture & & & $\checkmark$ & $\checkmark$ & 2 others \\
\hline Forestry & & & $\checkmark$ & & 2 others \\
\hline Legislation & $\checkmark$ & $\checkmark$ & $\checkmark$ & $\checkmark$ & 4 others \\
\hline Domestic households & & & & & 1 other \\
\hline Airports & & & & $\checkmark$ & \\
\hline Aquaculture & & & $\checkmark$ & & 2 others \\
\hline
\end{tabular}




\section{PROJECT OVERVIEW}




\section{Target Monitoring Stations}

\begin{tabular}{|c|c|c|c|c|c|c|c|}
\hline County & Site & Rationale & POCIS & PDMS & Water & Mussels & $\begin{array}{l}\text { Fish } \\
\text { (IFI) }\end{array}$ \\
\hline Cork & Inchigeelagh & Upstream river station & $\checkmark$ & $\checkmark$ & $\checkmark$ & & $\checkmark$ \\
\hline & Inniscarra & Downstream river station & $\checkmark$ & $\checkmark$ & $\checkmark$ & & $\checkmark$ \\
\hline & Shandon & Riverine/transitional & $\checkmark$ & $\checkmark$ & $\checkmark$ & & $\checkmark$ \\
\hline & Lough Mahon & Riverine/transitional station & $\checkmark$ & $\checkmark$ & $\checkmark$ & $\checkmark$ & \\
\hline & Outer bay & Riverine/transitional station & $\checkmark$ & $\checkmark$ & $\checkmark$ & $\checkmark$ & \\
\hline Dublın & Malahide & High pressure coastal & $\checkmark$ & $\checkmark$ & $\checkmark$ & $\checkmark$ & \\
\hline & Poolbeg & Riverine/transitional & $\checkmark$ & $\checkmark$ & $\checkmark$ & $\checkmark$ & \\
\hline & Lucan Bridge & Downstream river station & $\checkmark$ & $\checkmark$ & $\checkmark$ & & $\checkmark$ \\
\hline & Kilcullen Bridge & Upstream river station & $\checkmark$ & $\checkmark$ & $\checkmark$ & & $\checkmark$ \\
\hline Galway & |Kilkieran Bay & Coastal reference station & $\checkmark$ & $\checkmark$ & $\checkmark$ & $\checkmark$ & \\
\hline Mayo & Burrishoole & Upstream river station & $\checkmark$ & $\checkmark$ & $\checkmark$ & & $\checkmark$ \\
\hline \multirow[t]{2}{*}{ Donegal } & Glen Lackagh 1 & Cypermethrin study & SPMD & $\checkmark$ & $\checkmark$ & \multirow{2}{*}{\multicolumn{2}{|c|}{$\begin{array}{c}\text { EPA Benthic kick } \\
\text { sampling }\end{array}$}} \\
\hline & Glen Lackagh 2 & Cypermethrin study & SPMD & $\checkmark$ & $\checkmark$ & & \\
\hline
\end{tabular}




\section{Catchment Approach}

- The WFD introduced a comprehensive catchment based approach to water management

- Identify point sources and pathways of pollution

- More targeted approach to monitoring of emerging and priority compounds in water

- Potential role for the combination of catchment based approaches and focused water and passive sampler analysis for the surveillance monitoring 


\section{PHARMACEUTICALS STUDY}




\section{Cork 2013-2014}

- Method development

- LC-MS/MS

- GC-MS/MS

- PS deplovment
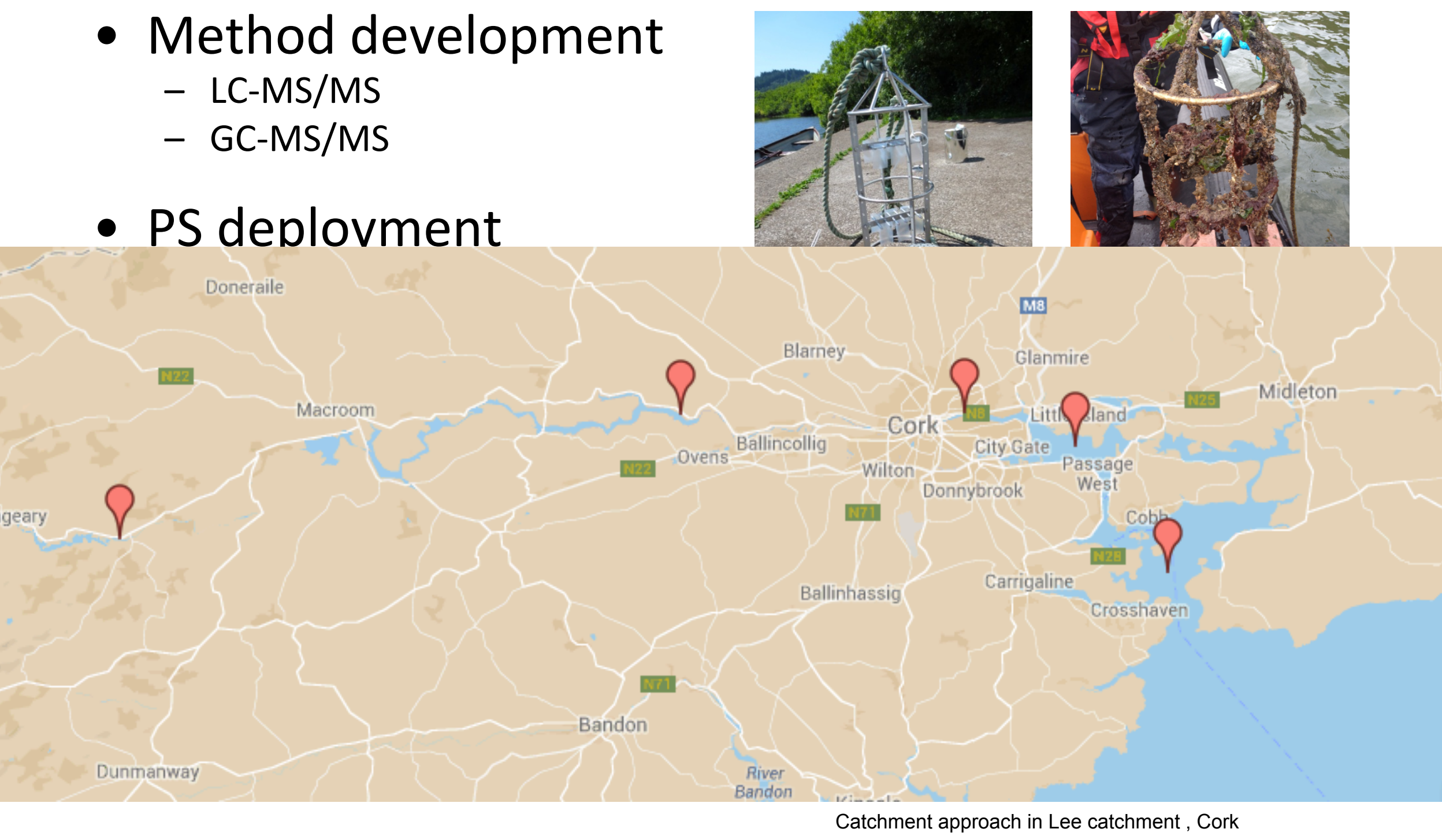


\section{Cork POCIS and water oestrogens}

\section{Upstream}

\begin{tabular}{|c|c|c|c|c|c|c|c|}
\hline & Matrix & & $\begin{array}{l}\text { Lough Allua } \\
\text { Inchigeelagh }\end{array}$ & Iniscarra & Shandon & $\begin{array}{l}\text { Lough } \\
\text { Mahon }\end{array}$ & $\begin{array}{c}\text { Cork Outer } \\
\text { Harbour }\end{array}$ \\
\hline Analyte & & Units & \multicolumn{5}{|c|}{2013} \\
\hline EE2 & \multirow{2}{*}{ POCIS } & $\mathrm{ng} \mathrm{L}^{-1}$ & $<0.2$ & 1.39 & $<0.2$ & $<0.2$ & $<0.2$ \\
\hline E2 & & $n g L^{-1}$ & $<0.5$ & $<0.5$ & $<0.5$ & 2.36 & 1.98 \\
\hline EE2 & \multirow{2}{*}{ Water } & $\mathrm{ng} \mathrm{L}^{-1 *}$ & nd & nd & nd & nd & nd \\
\hline E2 & & $\mathrm{ng} \mathrm{L}^{-1 *}$ & nd & nd & nd & nd & nd \\
\hline Analyte & & Units & \multicolumn{5}{|c|}{2014} \\
\hline E1 & \multirow{3}{*}{ POCIS } & $n g L^{-1}$ & $<0.51$ & 0.24 & 0.37 & 0.48 & 0.37 \\
\hline EE2 & & $\mathrm{ng} \mathrm{L}^{-1}$ & $<0.12$ & $<0.04$ & $<0.04$ & $<0.04$ & 0.07 \\
\hline E2 & & $\mathrm{ng} \mathrm{L}^{-1}$ & $<0.13$ & $<0.04$ & $<0.04$ & 0.06 & 0.09 \\
\hline E1 & \multirow{3}{*}{ Water } & $\mathrm{ng} \mathrm{L}-1 *$ & nd & 0.41 & nd & 0.41 & 0.54 \\
\hline EE2 & & $\mathrm{ng} \mathrm{L}-1 *$ & nd & nd & nd & nd & nd \\
\hline E2 & & $\mathrm{ng} \mathrm{L}^{-1 *}$ & nd & nd & nd & nd & nd \\
\hline
\end{tabular}

*LOD water samples by LC-MS/MS: E1: $0.07 \mathrm{ng} \mathrm{L}^{-1} \mathrm{E2}: 0.07 \mathrm{ng} \mathrm{L}^{-1}$, EE2, $0.11 \mathrm{ng} \mathrm{L}^{-1} .5 \mathrm{~L}$ sample $\mathrm{n}=2$ Effective sampling rates POCIS (ng/sampler/day)*: E1: 0.39, E2: 0.46, EE2: 0.235 


\section{Results: POCIS Temporal aspects}

- Due to issues with variability of environmental conditions in the field it was necessary to evaulate deployment duration for POCIS

-4 week deployment has been deemed the optimal timeframe for POCIS deployment

\begin{tabular}{|l|c|c|c|c|}
\hline & & \multicolumn{3}{|c|}{ Shandon Boat Club } \\
\hline Analyte & $\begin{array}{c}\text { Estimated } \\
\text { Water Conc. }\end{array}$ & 2 Weeks & 4 Weeks & 6 Weeks \\
\hline $\begin{array}{l}\text { 17alpha } \\
\text { ethynylestradiol } \\
\text { (EE2) }\end{array}$ & $\mathrm{ng} \mathrm{L}-1$ & $<0.09$ & 0.07 & $<0.04$ \\
\hline 17beta estradiol (E2) & $\mathrm{ng} \mathrm{L}^{-1}$ & $<0.10$ & 0.09 & 0.08 \\
\hline Estrone (E1) & $\mathrm{ng} \mathrm{L}^{-1}$ & $<0.39$ & 0.37 & 0.29 \\
\hline
\end{tabular}




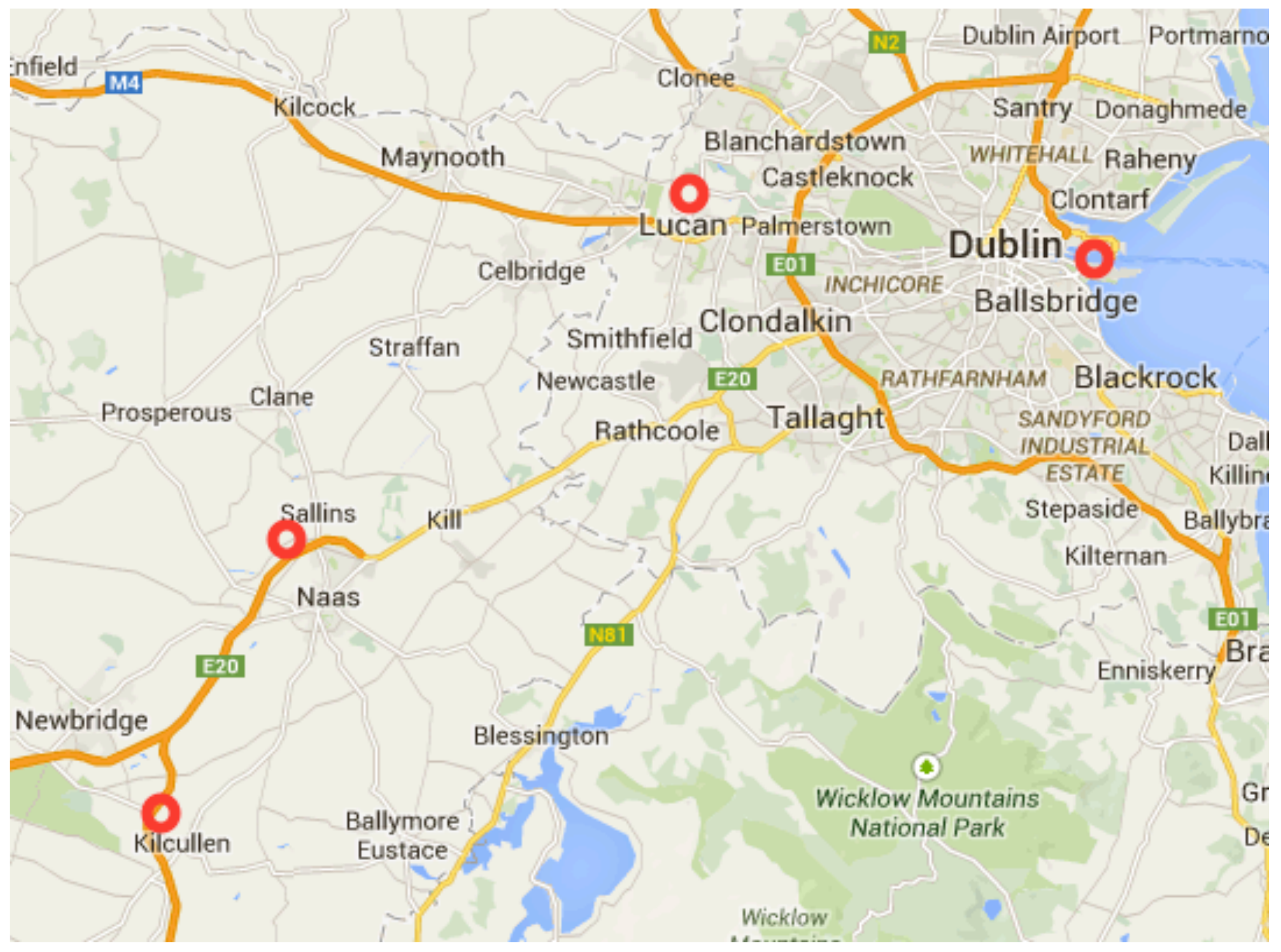




\section{Results: Estrogens in water}

\begin{tabular}{|c|c|c|c|}
\hline Analyte & E1 ng L$^{-1}$ & E2 ng L $^{-1}$ & EE2 g L $^{-1}$ \\
\hline Kilcullen T0 & $<0.07$ & nd & nd \\
\hline Kilcullen T1 & nd & nd & nd \\
\hline Osberstown T0 & nd & 0.33 & nd \\
\hline Osberstown T1 & nd & nd & nd \\
\hline Lucan Bridge T0 & 0.33 & 0.43 & nd \\
\hline Lucan Bridge T1 & nd & nd & nd \\
\hline Poolbeg T0 & nd & $<0.07$ & nd \\
\hline Poolbeg T1 & 1.92 & 0.23 & nd \\
\hline
\end{tabular}

T0 = Time zero, $\mathrm{T} 1$ = one month after sampler deployment

LOD water samples by LC-MS/MS: E1: $0.07 \mathrm{ng} / \mathrm{L} \mathrm{E2:} 0.07 \mathrm{ng} / \mathrm{L}, \mathrm{EE} 2,0.11 \mathrm{ng} / \mathrm{L} .5 \mathrm{~L}$ sample $\mathrm{n}=2$ 


\section{Environmental challenges and solutions}

- PS addresses challenges of detecting at low EQS

- Dissolved vs total water concentration remains an issue

- Time-integrated measurements

- Easy to deploy and analyse

- Simpler matrix

- Lack of confounding biological factors

- Suitable for "temporal" trend monitoring (and for surveillance/ screening) and for co-deployment with biota

- Ongoing development of modelling and partition coefficients will drive capabilities 


\section{Outputs from research}

- Comprehensive review of PS as a support to WFD monitoring

- Assessment of PS as surrogate to biota

- Assessment of priority pollutants in Irish catchments

- Recommendations for use of PS as a screening/monitoring tool 


\section{Project Media}

- Twitter: @irishPSresearch

- Website: https://sites.google.com/site/irishpassive sampling/home

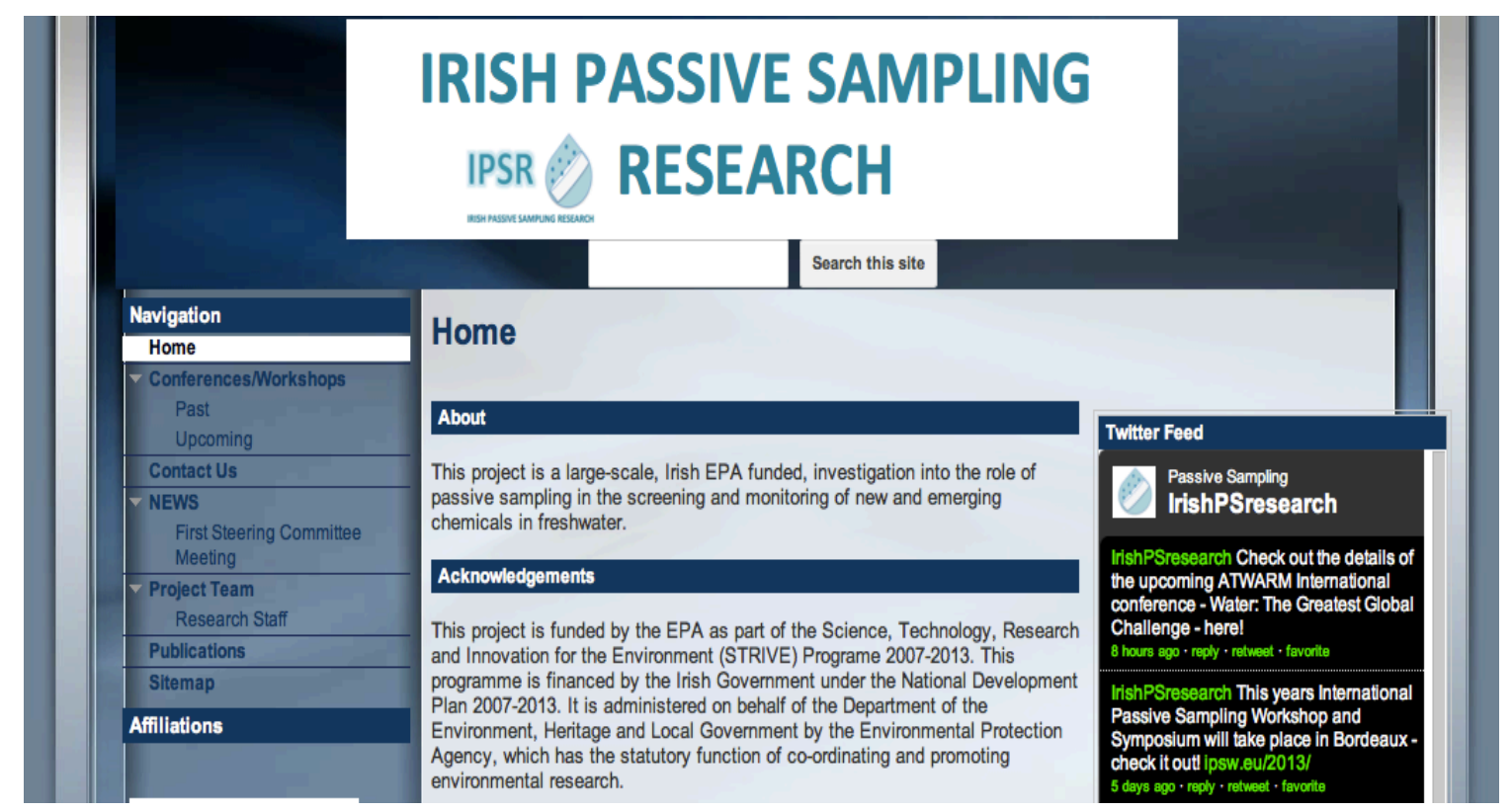




\section{Acknowledgements}

This project is funded by the EPA as part of the Science, Technology, Research and Innovation for the Environment (STRIVE) Programme 2007-2013. This programme is financed by the Irish Government under the National Development Plan 2007-2013.

It is administered on behalf of the Department of the Environment, Heritage and Local Government by the Environmental Protection Agency, which has the statutory function of co-ordinating and promoting environmental research.
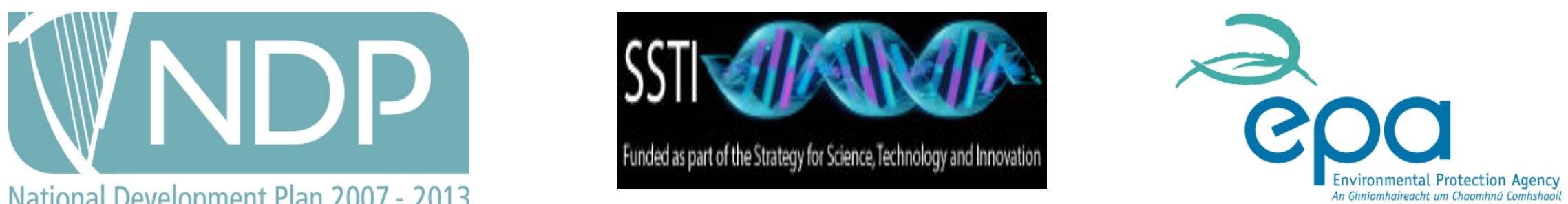